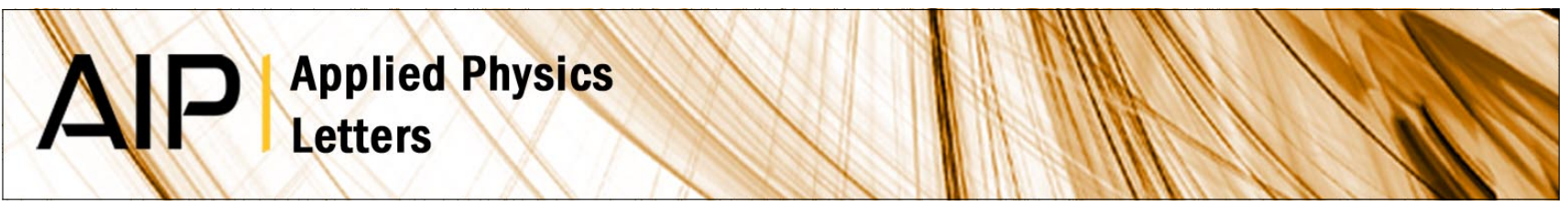

\title{
Centimeter scale atomic force microscope imaging and lithography
}

S. C. Minne, J. D. Adams, G. Yaralioglu, S. R. Manalis, A. Atalar et al.

Citation: Appl. Phys. Lett. 73, 1742 (1998); doi: 10.1063/1.122263

View online: http://dx.doi.org/10.1063/1.122263

View Table of Contents: http://apl.aip.org/resource/1/APPLAB/v73/i12

Published by the American Institute of Physics.

\section{Related Articles}

Bias controlled capacitive driven cantilever oscillation for high resolution dynamic force microscopy Appl. Phys. Lett. 102, 073110 (2013)

Friction measurement on free standing plates using atomic force microscopy

Rev. Sci. Instrum. 84, 013702 (2013)

A correlation force spectrometer for single molecule measurements under tensile load J. Appl. Phys. 113, 013503 (2013)

Compact metal probes: A solution for atomic force microscopy based tip-enhanced Raman spectroscopy Rev. Sci. Instrum. 83, 123708 (2012)

Note: Radiofrequency scanning probe microscopy using vertically oriented cantilevers

Rev. Sci. Instrum. 83, 126103 (2012)

\section{Additional information on Appl. Phys. Lett.}

Journal Homepage: http://apl.aip.org/

Journal Information: http://apl.aip.org/about/about_the_journal

Top downloads: http://apl.aip.org/features/most_downloaded

Information for Authors: http://apl.aip.org/authors

\section{ADVERTISEMENT}

\section{AIP Applied Physics Letters}

\section{EXPLORE WHAT'S NEW IN APL}

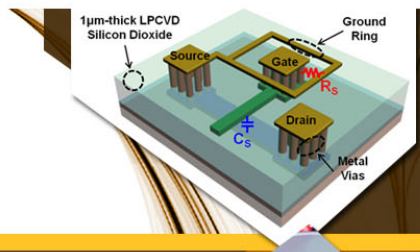

SURFACES AND INTERFACES

Focusing on physical, chemical, biological structural, optical, magnetic and electrical properties of surfaces and interfaces, and more.. 


\title{
Centimeter scale atomic force microscope imaging and lithography
}

\author{
S. C. Minne, ${ }^{\text {a) }}$ J. D. Adams, G. Yaralioglu, S. R. Manalis, A. Atalar, and C. F. Quate \\ E. L. Ginzton Laboratory, Stanford University, Stanford, California 94305-4085
}

(Received 18 May 1998; accepted for publication 10 July 1998)

\begin{abstract}
We present a $4 \mathrm{~mm}^{2}$ image taken with a parallel array of 10 cantilevers, an image spanning $6.4 \mathrm{~mm}$ taken with 32 cantilevers, and lithography over a $100 \mathrm{~mm}^{2}$ area using an array of 50 cantilevers. All of these results represent scan areas that are orders of magnitude larger than that of a typical atomic force microscope $\left(0.01 \mathrm{~mm}^{2}\right)$. Previously, the serial nature and limited scan size of the atomic force microscope prevented large scale imaging. Our design addresses these issues by using a modular micromachined parallel atomic force microscope array in conjunction with large displacement scanners. High-resolution microscopy and lithography over large areas are important for many applications, but especially in microelectronics, where integrated circuit chips typically have nanometer scale features distributed over square centimeter areas. (C) 1998 American Institute of Physics. [S0003-6951(98)04636-1]
\end{abstract}

The search for nonoptical lithographic systems used to pattern $0.1 \mu \mathrm{m}$ features is a daunting task, but as Fowler ${ }^{1}$ points out in his Physics Today article on the subject, "If there is no attempt to find alternatives, they will never be found." An array of scanning probes is one attempt to find the alternative for future systems.

The scanning probe is a tool for imaging and writing. The tips have been used to image atomic sized pixels, and to write sub- $0.1 \mu \mathrm{m}$ lines in positive and negative resists. But as it stands now, for wafer scale operation, the throughput is unacceptable. It is clear that the single tip scanning at modest speeds must be replaced with arrays of tips scanning at high speeds. Each tip in the array must operate independently to read, or write, individual pixels on demand.

Several groups are pursuing the parallel probe approach for imaging and surface modification. Lutwyche et al., ${ }^{2}$ Chui et al. ${ }^{3}$ and Ried et $a l .{ }^{4}$ have developed arrays of piezoresistive cantilevers, used them in parallel imaging applications, and have applied this technology to surface modification for data storage. Lang et al. ${ }^{5}$ have developed parallel arrays of optical probe devices for imaging and biological applications. Manalis ${ }^{6}$ has developed a parallel interferometric probing system that shows promise due to its sensitivity, simplicity, and expandability.

Device oriented applications of scanning probe lithography are also being pursued in many laboratories. ${ }^{7-10}$ Additionally, resist systems that use fast scanning $^{11}$ and parallelism ${ }^{12}$ are being developed to address lithography throughput issues.

In this letter we report on our recent progress in imaging and performing lithography with cantilever arrays. The various surface areas for the imaging and lithography data presented here are orders of magnitude larger than that of a typical atomic force microscope. This improvement is important because the areas imaged and patterned are commensurate with the areas of typical integrated circuit chips (100 $\mathrm{mm}^{2}$ ).

A description of the cantilever array and the electronic

${ }^{a)}$ Electronic mail: sminne@ee.stanford.edu system used in this work has been published previously. ${ }^{13}$ One centimeter arrays (50 cantilevers on a $200 \mu \mathrm{m}$ period) are microfabricated with integrated piezoresistive sensors and integrated zinc-oxide $(\mathrm{ZnO})$ actuators. The piezoresistive sensors provide 35 A resolution in a $20 \mathrm{kHz}$ bandwidth. More important, the integrated sensor simplifies the operation of the array because it requires no external components or alignment. The operation of the integrated sensor and actuator is automated with PC controlled analog circuitry. Details of the custom electronics and instrumentation configuration can be found in the aforementioned Ref. 13.

Figure 1 displays a $2 \mathrm{~mm} \times 2 \mathrm{~mm}$ atomic force microscope (AFM) image of a memory cell on an integrated circuit taken with 10 cantilevers operating in parallel. Each of the 10 cantilevers swept out a vertical swath $200 \mu \mathrm{m} \times 2 \mathrm{~mm}$, with a pixel density of $512 \times 5120$. This corresponds to a pixel size of roughly $0.4 \mu \mathrm{m}$. The pixel density of this image is not a fundamental limitation of the system or the data acquisition bandwidth, but arises due to technical limitations in processing the large amounts of data. The raw composite image size is over 100 Mbytes. The inset of Fig. 1 shows details contained within the acquired data that are not visible in the larger presentation. It should be noted that this area was not rescanned, but rather the data were simply extracted from the larger file.

In the composite presentation the color tables vary from image to image. This is primarily due to the autoscaling that is done for the image display. Currently, the maximum and minimum pixel values define the span of the color table, however, particles and defects, which are not uniform on each swath, generally dominate these extremes. For a more uniform presentation, average and deviation should determine the swath's contrast. While this does not affect the dimensional data, each tip's sensitivity and shape should be calibrated to ensure the accuracy of the measurement.

The inset of Fig. 1 also spans the seam between the adjacent tip's imaging paths. By scanning over a distance greater than array period $(200 \mu \mathrm{m})$, the adjacent tip's images can be stitched together to form a full fill image. Conventional piezotubes are not suitable for this type of scanning 


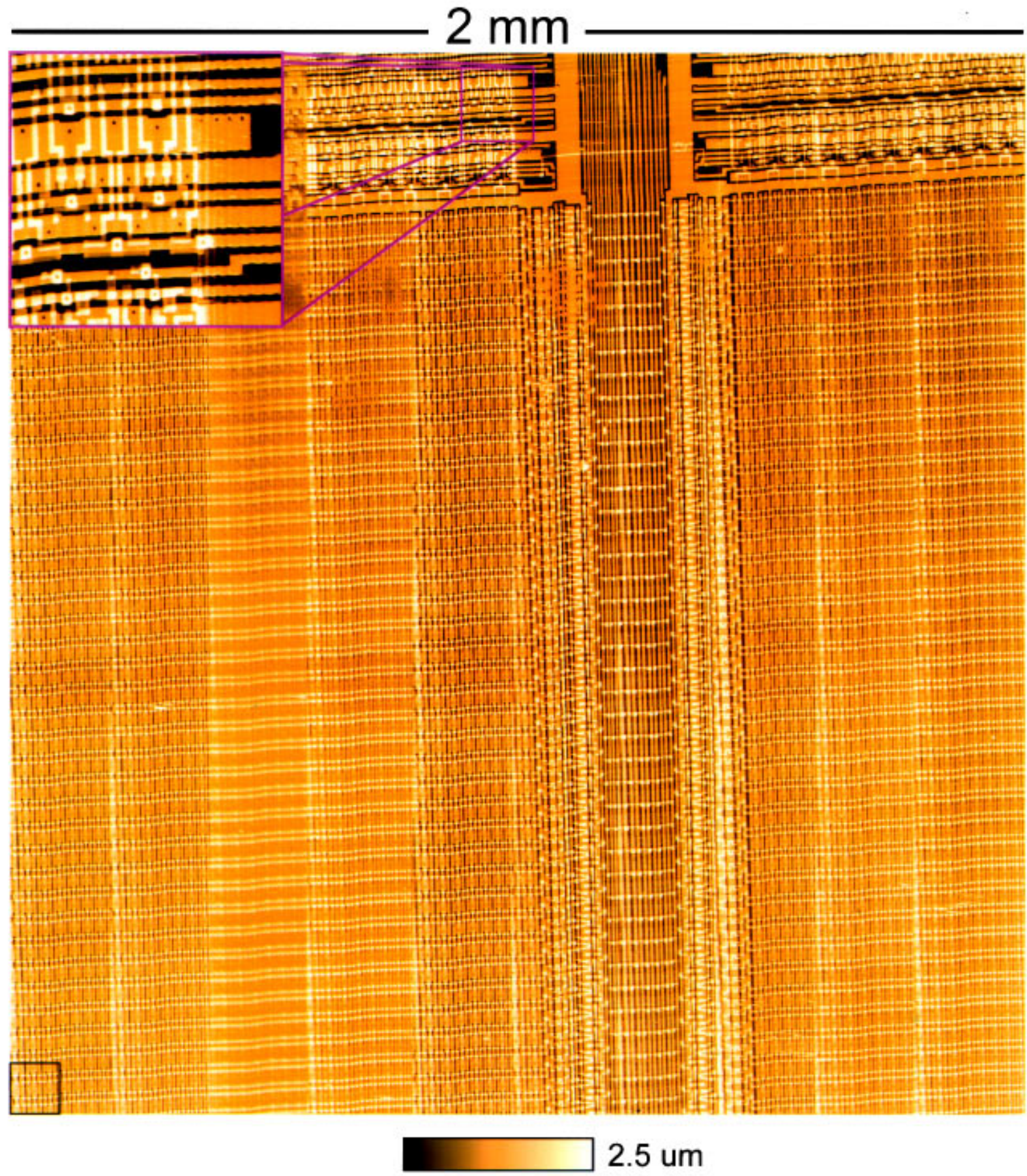

FIG. 1. $2 \mathrm{~mm} \times 2 \mathrm{~mm}$ AFM image of an integrated circuit chip. The image was acquired with 10 cantilevers in parallel, where each cantilever imaged a vertical swath one-tenth of the composite image size. The horizontal scan size was $200 \mu \mathrm{m}$ (the tip to tip spacing), the vertical scan size was $2 \mathrm{~mm}$, the tip speed was $1 \mathrm{~mm} / \mathrm{s}$, and the pixel size was $0.4 \mu \mathrm{m}$. The entire image was acquired in $30 \mathrm{~min}$. The box at the bottom left-hand side of the image represents the maximum scan size of $100 \mu \mathrm{m} \times 100 \mu \mathrm{m}$ from a typical AFM.

due to their $z$-axis coupling. To solve this problem a custom flexure scanner (Nikon) was mounted on a long-range highresolution scanner (Newport PM-500). The image was acquired at $1 \mathrm{~mm} / \mathrm{s}(2.5 \mathrm{~Hz}$ over $200 \mu \mathrm{m})$.

Figure 2 shows an image that spans $6.4 \mathrm{~mm}$ and was taken with 32 cantilevers operating in parallel. Again, since the scan distance exceeded the tip period, a full fill representation of the surface is obtained. The sample is a twodimensional grating with a period of $20 \mu \mathrm{m}$ and a step height of $200 \mathrm{~nm}$. The image has been broken into four strips and offset vertically for display purposes. The cantilevers were controlled by two custom-built circuit boards interfaced to a PC. Each board contains the circuitry necessary to control 16 cantilevers. We believe this represents the largest parallel probe imaging operation to date. The small box in the corner of Fig. 2 represents the maximum scan of a typical AFM.

High-resolution imaging of large areas is useful in many applications. Furthermore, probe-based imaging also represents a starting point for probe-based lithography. The imaging capabilities in a lithography system are useful for inspec- 


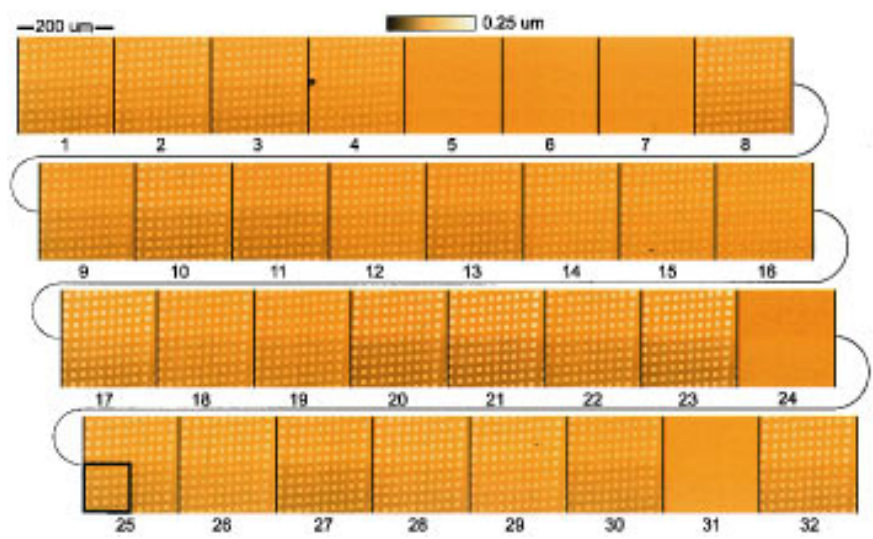

FIG. 2. A $32 \times 1$ parallel AFM image of a two-dimensional diffraction grating. The horizontal image distance is $6.4 \mathrm{~mm}$; the entire image area is 1.28 $\mathrm{mm}^{2}$. The image represents the horizontal combination of the 32 individual images. It has been broken into four strips, and offset vertically, for display purposes. The image was taken in the same manner as Fig. 1. The box at the bottom left-hand corner of the image represents the maximum scan size of a typical AFM, $100 \mu \mathrm{m} \times 100 \mu \mathrm{m}$.

tion of fabricated patterns, but more important, imaging capabilities represent the ability to lithographically overlay to preexisting patterns.

Figure 3 shows a $1 \mathrm{~cm}^{2}$ area patterned with a single pass

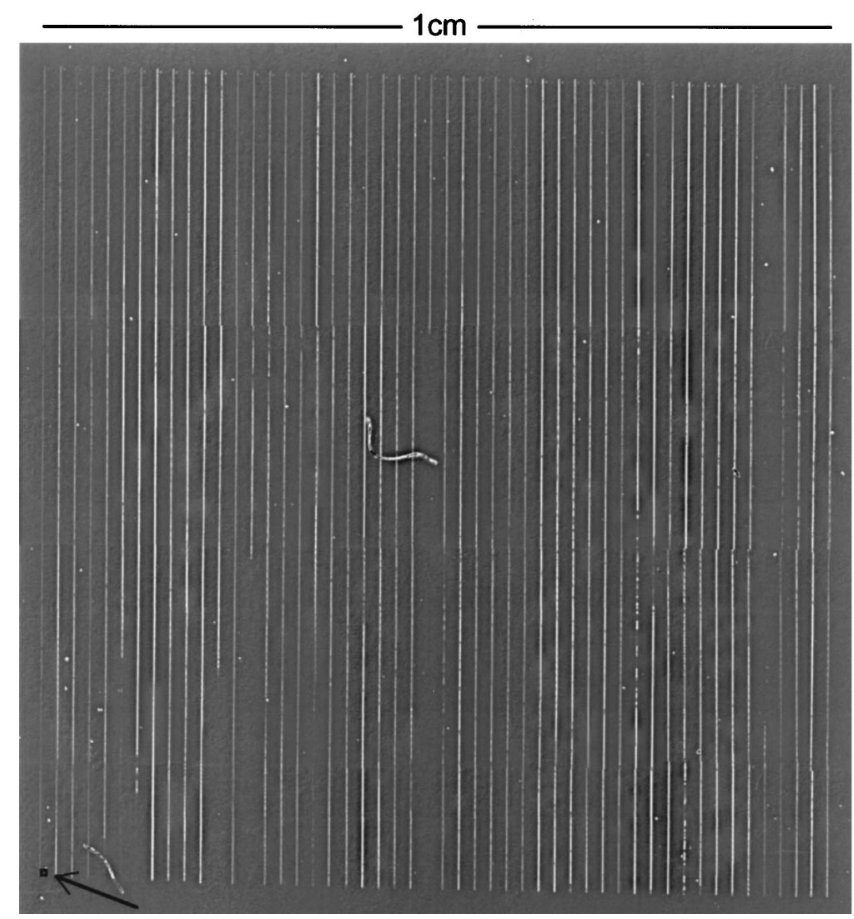

FIG. 3. $50 \times 1$ parallel AFM lithography over $1 \mathrm{~cm}^{2}$. The lithography was accomplished by electric field enhanced oxidation of silicon at $15 \mathrm{~V}$, and at a scan speed of $1 \mu \mathrm{m} / \mathrm{s}$. The lithographed oxide pattern was transferred into the silicon using $\mathrm{KOH}$. The small box at the bottom left-hand side of the image represents the maximum scan size of a typical AFM, $100 \mu \mathrm{m} \times 100$ $\mu \mathrm{m}$. The picture was formed from 24 optical photographs taken with a $5 \times$ Nomarski microscope. of a 50 cantilever array. The substrate was (100) hydrogen passivated silicon. The intense electric field resulting from the voltage applied between the tips and sample $(15 \mathrm{~V})$ anodizes the silicon, resulting in thin oxide lines. This thin oxide was then used as an etch mask for transferring the pattern to the silicon substrate in $\mathrm{KOH}$. Detailed discussions of the anodization process are found elsewhere. ${ }^{7,8,10,12}$ The tip speed during lithography was $1 \mu \mathrm{m} / \mathrm{s}$.

The transferred linewidths are on average $1.1 \mu \mathrm{m}$. In the displayed image, the patterned lines are artificially widened from the image acquisition process. The $1 \mathrm{~cm}^{2}$ image was formed from 24 optical photographs taken from a microscope operating in the Nomarski interference contrast (NIC) mode. The NIC and the subsequent digitization of the resulting photographs cause displayed linewidths to appear broader than they really are.

In conclusion, the results presented here represent scan areas that are orders of magnitude larger than that of a typical atomic force microscope $\left(0.01 \mathrm{~mm}^{2}\right)$. These results are an essential step to covering even larger scan areas with increased throughput for both imaging and lithography. Work is currently underway to expand the size and functionality of the cantilever arrays, and the associated mechanical and electrical hardware, in order to operate in feedback mode over large areas.

The authors would like to thank Nick Ulman and Jim Zesch for their insightful comments. S.R.M. acknowledges the support of a Leland T. Edwards fellowship, and J.D.A. acknowledges the support of a NSF graduate fellowship. This work was supported by the Defense Advanced Research Projects Agency and the Semiconductor Research Corporation.

${ }^{1}$ A. Fowler, Phys. Today 50, 50 (1997).

${ }^{2}$ M. Lutwyche, C. Andreoli, G. Binnig, J. Brugger, U. Drechsler, W. Haeberle, H. Rohrer, H. Rothuizen, and P. Vettiger, Proceedings of the IEEE International Workshop on Microelectro Mechanical Systems (MEMS 98), Heidelberg, Germany, 25-29 January 1998.

${ }^{3}$ B. W. Chui, T. D. Stowe, T. W. Kenny, H. J. Mamin, B. D. Terris, and D. Rugar, Appl. Phys. Lett. 69, 2767 (1996).

${ }^{4}$ R. P. Ried, H. J. Mamin, B. D. Terris, L. S. Fan, and D. Rugar, J. Microelectromech. 6, 294 (1997).

${ }^{5}$ H. P. Lang, R. Berger, C. Andreoli, J. Brugger, M. Despont, P. Vettiger, Ch. Gerber, J. K. Gimzewski, J. P. Ramseyer, E. Meyer, and H. J. Guntherodt, Appl. Phys. Lett. 73, 383 (1998).

${ }^{6}$ S. R. Manalis, S. C. Minne, A. Atalar, and C. F. Quate, Appl. Phys. Lett. 69, 3944 (1996).

${ }^{7}$ E. S. Snow and P. M. Campbell, Science 270, 1639 (1995).

${ }^{8}$ K. Matsumoto, M. Ishii, J. Shirakashi, B. J. Vartanian, and J. S. Harris, Proceedings of Quantum Devices and Circuits, Alexandria, Egypt, 4-7 June 1996 (unpublished).

${ }^{9}$ H. T. Soh, K. Wilder, A. Atalar, and C. F. Quate, Proceedings of the 1997 Symposium on VLSI Technology, Kyoto, Japan, pp. 129 and 130.

${ }^{10}$ S. C. Minne, H. T. Soh, Ph. Flueckiger, and C. F. Quate, Appl. Phys. Lett. 6, 703 (1995).

${ }^{11}$ S. W. Park, H. T. Soh, C. F. Quate, and S.-I. Park, Appl. Phys. Lett. 67, 2415 (1995)

${ }^{12}$ S. C. Minne, S. R. Manalis, A. Atalar, and C. F. Quate, J. Vac. Sci. Technol. B 14, 2456 (1996).

${ }^{13}$ S. C. Minne, G. Yaralioglu, S. R. Manalis, J. D. Adams, J. Zesch, A. Atalar, and C. F. Quate, Appl. Phys. Lett. 72, 2340 (1998). 